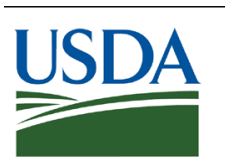

United States

Department of

Agriculture

\title{
Brazil Soybean Transportation
}

\section{A quarterly publication of the Agricultural Marketing Service \\ www.ams.usda.gov/services/transportation-analysis}

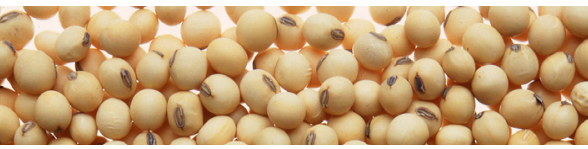

First Quarter 2020 (July, August, September) Published May 27, 2020

\section{Weakened Currency Spurs Record Exports, Variable Transportation Costs, and Higher Domestic Prices}

From first quarter 2019 to first quarter 2020 (year to year), the Brazilian real depreciated nearly 19 percent against the U.S. dollar, from R\$3.77 per U.S. dollar to R\$4.47 per U.S. dollar (Brazil Central Bank). As of April 30, 2020, year-to-date exports reached a record of 33.7 million metric tons ( $\mathrm{mmt}$ ) valued at $\$ 11.5$ billion, about 34 percent more than at this time last year (Comex Stat, Ministério da Indústria, Comércio Exterior e Serviços (MDIC)). Year to year, the cost of shipping a metric ton (mt) of soybeans 100 miles by truck decreased 18 percent from $\$ 7.75$ per $\mathrm{mt}$ to $\$ 6.63$ per $\mathrm{mt}$ (table 8 ). This reduction reflects the weakening of the Brazilian real (R\$) against the U.S. dollar.

Figure 1. Southern ports exported 63 percent of Brazilian soybeans, January-April 2020

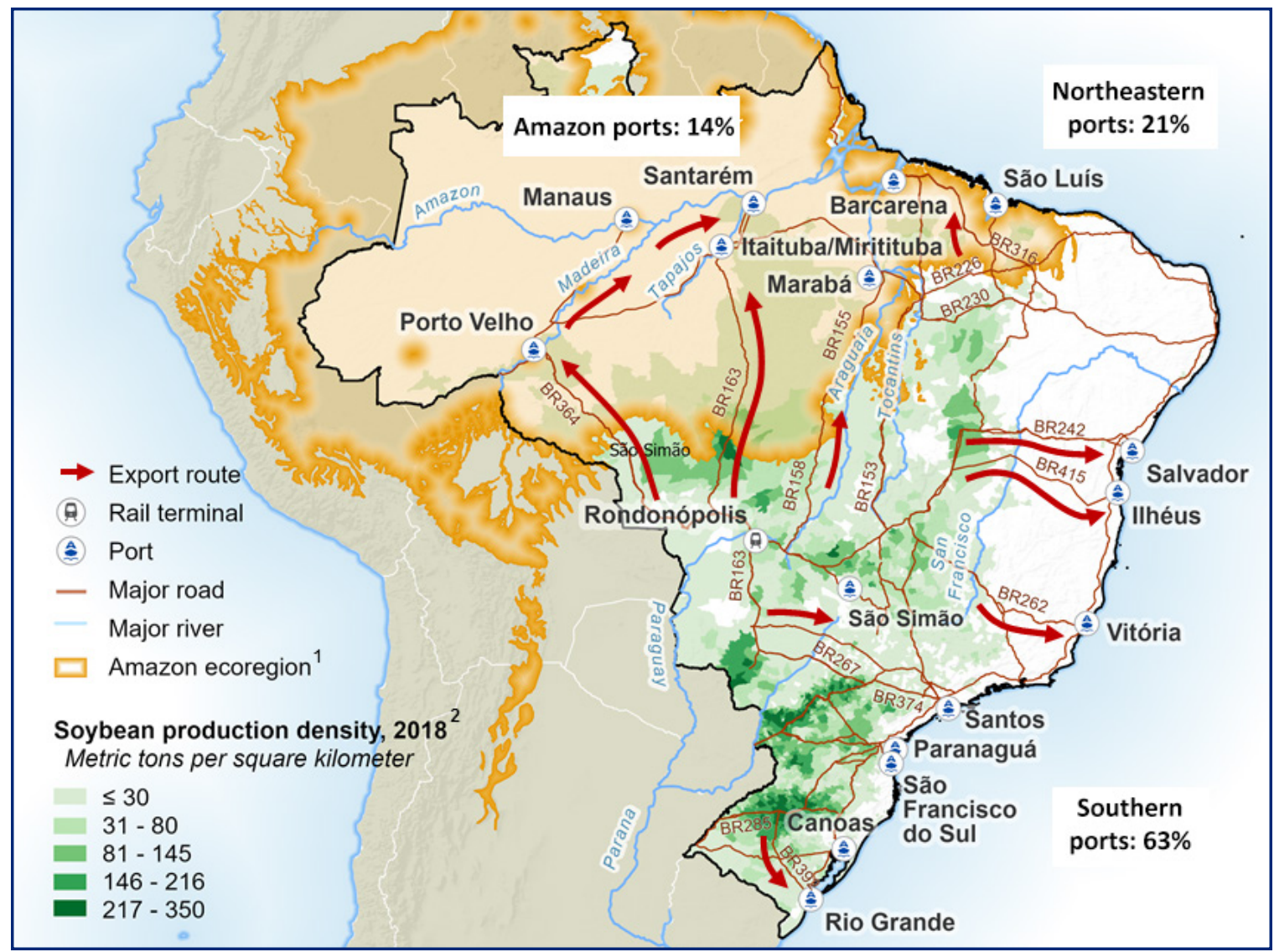

1 World Wildlife Fund.

2 Brazilian Institute of Geography and Statistics-Produção Agricola Municipal.

Source: USDA/Agricultural Marketing Service and USDA/Foreign Agricultural Service. 


\section{Brazil Soybean Transportation}

However, truck rates measured in reais $(\mathrm{R} \$)$ varied by region from their estimates in U.S. dollars largely because of improvements in transportation infrastructure. In particular, the completion of BR 163 (the major route connecting Sorriso, North Mato Grosso, to Itaituba, Pará) significantly contributed to the variance in regional rates. Finishing the project expedited deliveries of soybean exports via the river ports of Barcarena $(2.8 \mathrm{mmt})$, Santarém $(2.1 \mathrm{mmt})$, and Manaus $(1.5 \mathrm{mmt})$. Over the last 5 years, Barcarena soybean exports nearly quadrupled, from $1.1 \mathrm{mmt}$ in 2014 to $5.8 \mathrm{mmt}$ in 2019. As of April 30, 2020, Barcarena was the thirdlargest Brazilian soybean exporting port after Santos $(11.8 \mathrm{mmt})$ and Paranaguá. $(5.7 \mathrm{mmt})$. All these factors combined to contribute to regional rate variances year to year. For example, truck rates from Cruz Alta, Rio Grande do Sul to Rio Grande, increased 4 percent. In contrast, truck rates from North Mato Grosso to Rondonópolis (rail terminal) decreased 2 percent and 12-18 percent to the northern inland ports of Santarém and Itaituba/Miritituba (barge terminal), respectively. Intensified competition from the northbound route decreased truck rates from Sorriso, North Mato Grosso to the southern port of Santos about 2 percent although rates from Sorriso, North Mato Grosso to Paranaguá increased 12 percent.

In contrast, ocean freight costs in 2020 increased but not by enough to offset lower truck costs. For first quarter 2020, ocean rates declined overall, but remained higher than first-quarter 2019 levels. $^{3}$ On average, ocean rates from the southern Brazilian ports increased nearly 13 percent to Shanghai, China, year to year, compared to an increase of 29 percent to Hamburg, Germany (tables 1, 1a, and 9). In first quarter 2020, the ocean freight spread was $\$ 3 / \mathrm{mt}$ for the routes from the northeastern port of Barcarena $(\$ 38.50 / \mathrm{mt})$ and the port of Santos $(\$ 35.50)$ to Shanghai (table 9). ${ }^{4}$ The ocean freight spread was $\$ 1.25 / \mathrm{mt}$ for the routes from the northeastern port of São Luís $(\$ 36.75 / \mathrm{mt})$ and the port of Santos $(\$ 35.50)$ to Shanghai (table 9). In Sorriso, North MT-the largest Brazilian soybean-producing state-first quarter 2020 transportation costs represented nearly 26-27 percent of the total landed costs of shipping soybeans to Shanghai through Santos. Also, in Sorriso, transportation costs represented 22 percent of the total landed costs through the port of Santarém (tables 1 and 1a).

For the first 4 months of 2020, Santos was the largest Brazilian soybean export port, followed in descending order by Paranaguá, Barcarena, Rio Grande, São Luís, Santarém, São Francisco do Sul, and Manaus (fig. 1) (MDIC). These ports accounted for 93 percent of Brazil's total soybean exports. With the shares broken down from a north/south perspective, the southern ports of Santos, Rio Grande, Paranaguá, and São Francisco do Sul dominated the soybean trade, accounting for about 63 percent of Brazil's soybean exports. Meanwhile, the northeastern ports of São Luís, Vitória, Salvador, and Barcarena accounted for 21 percent of soybean exports. The Amazon River ports of Manaus and Santarém exported 14 percent.

Year to year, average 2020 Brazilian soybean export prices decreased 6 percent, from $\$ 358$ per mt to $\$ 335$ per $\mathrm{mt}(\mathrm{MDIC})$. The weakening of the Brazilian real against the U.S. dollar offset the slight year-to-year fall in farm gate prices, from $\$ 299.06 / \mathrm{mt}$ to $\$ 296.27 / \mathrm{mt}$, when measured in U.S. dollars because soybeans are priced in U.S. dollars but paid in reais (Companhia Nacional de Abastecimento (CONAB)). Farm prices measured in reais increased an average of 11 percent, from $R \$ 1,127.44 / \mathrm{mt}$ in 2019 to $\mathrm{R} \$ 1,316.94$ in the first quarter of 2020 (CONAB).

From January-April 2020, soybean exports to China increased 32 percent from $18.6 \mathrm{mmt}$ to $24.7 \mathrm{mmt}$ (valued at \$8.4 billion) (MDIC). China received 73 percent of Brazil's total soybean exports (33.7 $\mathrm{mmt}$ ). The next highest shares of Brazil's soybean exports went (in declining order) to the Netherlands, Spain, Turkey,

3 Ocean during first half of 2018 were unusually low because of lower Chinese iron ore imports and the collapse of a mine-waste dam in southeastern Brazil that disrupted Brazilian iron supply (Grain Transportation Report (GTR)).

4 Ocean freight spread is the cost difference between two vessel routes to the same destination. 


\section{Brazil Soybean Transportation}

Thailand, Pakistan and Mexico. The southern ports of Santos, Paranaguá, Rio Grande, and São Francisco do Sul accounted for 80 percent of total soybean exports to China (fig. 1). The Northeastern ports of São Luís, Vitoria, Barcarena, and Salvador accounted for nearly 20 percent of Brazilian soybeans to China. The Northern ports of Santarém and Manaus represented less than 1 percent of exports to China. For more information, contact Delmy L. Salin at delmy.salin@ams.usda.gov.

Table 1. Quarterly costs of transporting Brazilian soybeans from the southern ports to Shanghai, China

\begin{tabular}{|c|c|c|c|c|c|c|}
\hline & $\begin{array}{c}2019 \\
1 s t \text { qtr. }\end{array}$ & $\begin{array}{c}2020 \\
1 \text { st qtr. }\end{array}$ & $\begin{array}{l}\text { \% Change } \\
2019-20\end{array}$ & $\begin{array}{l}2019 \\
1 \text { st qtr. }\end{array}$ & $\begin{array}{c}2020 \\
1 \text { st qtr. }\end{array}$ & $\begin{array}{c}\text { \% Change } \\
2019-20\end{array}$ \\
\hline & \multicolumn{3}{|c|}{$\begin{array}{c}\text { North } \mathrm{MT}^{1} \text { - Santos }{ }^{2} \text { by truck } \\
\text {-US\$/mt- }\end{array}$} & \multicolumn{3}{|c|}{$\begin{array}{c}\text { Northwest } \text { RS }^{1} \text { - Rio Grande } \\
\text {-US\$/mt- }\end{array}$} \\
\hline Truck & 81.92 & 68.33 & -16.6 & 26.05 & 22.92 & -12.0 \\
\hline Ocean & 32.25 & 35.50 & 10.1 & 31.58 & 37.00 & 17.2 \\
\hline Total transportation & 114.17 & 103.83 & -9.0 & 57.63 & 59.92 & 4.0 \\
\hline Farm gate price ${ }^{3}$ & 275.38 & 282.59 & 2.6 & 308.52 & 300.04 & -2.7 \\
\hline Landed cost & 389.54 & 386.43 & -0.8 & 366.15 & 359.97 & -1.7 \\
\hline \multirow[t]{2}{*}{ Transport $\%$ of landed cost } & 29.3 & 26.9 & -8.3 & 15.7 & 16.6 & 5.8 \\
\hline & \multicolumn{3}{|c|}{$\begin{array}{c}\text { North } \mathrm{MT}^{1} \text { - Santos }{ }^{2} \text { by rail } \\
\text {-US\$/mt- }\end{array}$} & \multicolumn{3}{|c|}{$\begin{array}{c}\text { North } \mathrm{MT}^{1} \text { - Paranaguá } \\
\text {-US\$/mt- }\end{array}$} \\
\hline Truck & 29.89 & 24.79 & -17.1 & 71.05 & 67.48 & -5.0 \\
\hline Rail $^{4}$ & 41.21 & 37.73 & -8.5 & - & - & - \\
\hline Ocean & 32.25 & 35.50 & 10.1 & 33.75 & 37.25 & 10.4 \\
\hline Total transportation & 103.36 & 98.02 & -5.2 & 104.80 & 104.73 & -0.1 \\
\hline Farm gate price ${ }^{3}$ & 275.38 & 282.59 & 2.6 & 275.38 & 282.59 & 2.6 \\
\hline Landed cost & 378.73 & 380.61 & 0.5 & 380.18 & 387.32 & 1.9 \\
\hline Transport \% of landed cost & 27.3 & 25.8 & -5.6 & 27.6 & 27.0 & -1.9 \\
\hline
\end{tabular}

${ }^{1}$ Producing regions: RS = Rio Grande Do Sul and MT= Mato Grosso.

${ }^{2}$ Export port.

${ }^{3}$ The source of the farm gate price is the Brazilian Government, Companhia Nacional de Abastecimento (CONAB).

${ }^{4}$ In Brazil, there are no public/official rail tariff rates. Rail rates can be up to 30 percent lower than truck rates, depending on volumes hauled and the terms of contracts signed between the railroad company and shippers.

Note: qtr. $=$ quarter. $\mathrm{mt}=$ metric ton .

Source: University of São Paulo, Escola Superior de Agricultura "Luiz de Queiroz," Brazil (ESALQ/ USP) and USDA, Agricultural Marketing Service. 


\section{Brazil Soybean Transportation}

Table 1a. Quarterly costs of transporting Brazilian soybeans from the northern and northeastern ports to Shanghai, China

\begin{tabular}{|c|c|c|c|c|c|c|}
\hline & $\begin{array}{c}2019 \\
1 \text { st qtr. }\end{array}$ & $\begin{array}{c}2020 \\
\text { 1st qtr. }\end{array}$ & $\begin{array}{c}\text { \% Change } \\
2019-20\end{array}$ & $\begin{array}{c}2019 \\
\text { 1st qtr. }\end{array}$ & $\begin{array}{c}2020 \\
1 \text { st qtr. }\end{array}$ & $\begin{array}{c}\text { \% Change } \\
2019-20\end{array}$ \\
\hline & \multicolumn{3}{|c|}{$\begin{array}{c}\text { North } \mathrm{MT}^{1} \text { - Santarém } \\
\text {-US\$/mt- }\end{array}$} & \multicolumn{3}{|c|}{$\begin{array}{c}\text { South } \text { MA }^{1} \text { - São Luís } \\
-U S \$ / m t-\end{array}$} \\
\hline Truck & 59.40 & 44.10 & -25.8 & 37.04 & 28.86 & -22.1 \\
\hline Ocean & 32.25 & 36.50 & 13.2 & 31.00 & 36.75 & 18.5 \\
\hline Total transportation & 91.65 & 80.60 & -12.1 & 68.04 & 65.61 & -3.6 \\
\hline Farm gate price ${ }^{3}$ & 275.38 & 282.59 & 2.6 & 298.43 & 300.23 & 0.6 \\
\hline Landed cost & 367.03 & 363.20 & -1.0 & 366.47 & 365.83 & -0.2 \\
\hline \multirow[t]{2}{*}{ Transport $\%$ of landed cost } & 25.0 & 22.2 & -11.1 & 18.6 & 17.9 & -3.4 \\
\hline & \multicolumn{3}{|c|}{$\begin{array}{c}\text { Southwest } \mathrm{PI}^{1} \text { - São Luís }{ }^{2} \\
-U S \$ / m t-\end{array}$} & \multicolumn{3}{|c|}{$\begin{array}{c}\text { North } \mathrm{MT}^{1} \text { - Barcarena }{ }^{2} \\
\text {-US\$/mt- }\end{array}$} \\
\hline Truck & 45.24 & 32.49 & -28.2 & 53.99 & 37.11 & -31.3 \\
\hline Barge $^{4}$ & - & - & - & 19.66 & 16.42 & -16.5 \\
\hline Ocean & 31.00 & 36.75 & 18.5 & 32.25 & 38.50 & 19.4 \\
\hline Total transportation & 76.24 & 69.24 & -9.2 & 105.90 & 92.03 & -13.1 \\
\hline Farm gate price ${ }^{3}$ & 292.96 & 302.03 & 3.1 & 275.38 & 282.59 & 2.6 \\
\hline Landed cost & 369.20 & 371.27 & 0.6 & 381.28 & 374.62 & -1.7 \\
\hline Transport $\%$ of landed cost & 20.7 & 18.6 & -9.7 & 27.8 & 24.6 & -11.6 \\
\hline
\end{tabular}

${ }^{1}$ Producing regions: $\mathrm{MT}=$ Mato Grosso, $\mathrm{PI}=$ Piauí, and MA = Maranhão.

${ }^{2}$ Export port.

${ }^{3}$ The source of the farm gate price is the Brazilian Government, Companhia Nacional de Abastecimento (CONAB).

${ }^{4}$ In Brazil, there are no public/official Barge rates. Barge rates can be up to 60 percent lower than truck rates, depending on volumes hauled and the terms of contracts signed between the barge company and shippers. The distance is in nautical miles.

Note: qtr. $=$ quarter. $\mathrm{mt}=$ metric ton .

Source: University of São Paulo, Escola Superior de Agricultura “Luiz de Queiroz," Brazil (ESALQ/ USP) and USDA, Agricultural Marketing Service. 


\section{Brazil Soybean Transportation}

Table 2. Quarterly costs of transporting Brazilian soybeans from the southern ports to Hamburg, Germany

\begin{tabular}{|c|c|c|c|c|c|c|}
\hline & $\begin{array}{c}2019 \\
1 s t \text { qtr. }\end{array}$ & $\begin{array}{c}2020 \\
1 \text { st qtr. }\end{array}$ & $\begin{array}{c}\text { \% Change } \\
2019-20\end{array}$ & $\begin{array}{c}2019 \\
1 \text { st qtr. }\end{array}$ & $\begin{array}{c}2020 \\
1 \text { st qtr. }\end{array}$ & $\begin{array}{c}\text { \% Change } \\
2019-20\end{array}$ \\
\hline & \multicolumn{3}{|c|}{$\begin{array}{l}\text { North } \mathrm{MT}^{1} \text { - Santos } \\
\text {-US\$/mt- }\end{array}$} & \multicolumn{3}{|c|}{$\begin{array}{c}\text { Northwest RS }{ }^{1} \text { - Rio Grande } \\
\text {-US\$/mt- }\end{array}$} \\
\hline Truck & 81.92 & 68.33 & -16.6 & 26.05 & 22.92 & -12.0 \\
\hline Ocean & 23.00 & 29.25 & 27.2 & 23.00 & 29.50 & 28.3 \\
\hline Total transportation & 104.92 & 97.58 & -7.0 & 49.05 & 52.42 & 6.9 \\
\hline Farm gate price ${ }^{3}$ & 275.38 & 282.59 & 2.6 & 308.52 & 300.04 & -2.7 \\
\hline Landed cost & 380.29 & 380.18 & 0.0 & 357.57 & 352.47 & -1.4 \\
\hline \multirow[t]{2}{*}{ Transport \% of landed cost } & 27.6 & 25.7 & -7.0 & 13.7 & 14.9 & 8.4 \\
\hline & \multicolumn{3}{|c|}{$\begin{array}{l}\text { North } \mathrm{MT}^{1}{ }^{-} \text {Santos } \\
\text {-US\$/mt- }\end{array}$} & \multicolumn{3}{|c|}{$\begin{array}{c}\text { North } \mathrm{MT}^{1} \text { - Paranaguá } \\
\text {-US\$/mt- }\end{array}$} \\
\hline Truck & 29.89 & 24.79 & -17.1 & 71.05 & 67.48 & -5.0 \\
\hline Rail $^{4}$ & 41.21 & 37.73 & -8.5 & - & - & - \\
\hline Ocean & 23.00 & 29.25 & 27.2 & 23.00 & 30.00 & 30.4 \\
\hline Total transportation & 94.11 & 91.77 & -2.5 & 94.05 & 97.48 & 3.6 \\
\hline Farm gate price ${ }^{3}$ & 275.38 & 282.59 & 2.6 & 275.38 & 282.59 & 2.6 \\
\hline Landed cost & 369.48 & 374.36 & 1.3 & 369.43 & 380.07 & 2.9 \\
\hline Transport $\%$ of landed cost & 25.5 & 24.5 & -3.8 & 25.5 & 25.6 & 0.7 \\
\hline
\end{tabular}

${ }^{1}$ Producing regions: RS = Rio Grande Do Sul and MT= Mato Grosso.

${ }^{2}$ Export port.

${ }^{3}$ The source of the farm gate price is the Brazilian Government, Companhia Nacional de Abastecimento (CONAB).

${ }^{4}$ In Brazil, there are no public/official rail tariff rates. Rail rates can be up to 30 percent lower than truck rates, depending on volumes hauled and the terms of contracts signed between the railroad company and shippers.

Note: qtr. = quarter. $\mathrm{mt}=$ metric ton.

Source: University of São Paulo, Escola Superior de Agricultura “Luiz de Queiroz," Brazil (ESALQ/ USP) and USDA, Agricultural Marketing Service. 


\section{Brazil Soybean Transportation}

Table 2a. Quarterly costs of transporting Brazilian soybeans from the northern and northeastern ports to Hamburg, Germany

\begin{tabular}{|c|c|c|c|c|c|c|}
\hline & $\begin{array}{c}2019 \\
1 \text { st qtr. }\end{array}$ & $\begin{array}{c}2020 \\
1 \text { st qtr. }\end{array}$ & $\begin{array}{c}\text { \% Change } \\
2019-20\end{array}$ & $\begin{array}{c}2019 \\
\text { 1st qtr. }\end{array}$ & $\begin{array}{c}2020 \\
1 \text { st qtr. }\end{array}$ & $\begin{array}{c}\text { \% Change } \\
2019-20\end{array}$ \\
\hline & \multicolumn{3}{|c|}{$\begin{array}{c}\text { North } \mathrm{MT}^{1} \text { - Santarém } \\
\text {-US\$/mt- }\end{array}$} & \multicolumn{3}{|c|}{$\begin{array}{c}\text { South } \mathrm{MA}^{1} \text { - São Luís } \\
\text {-US\$/mt- }\end{array}$} \\
\hline Truck & 59.40 & 44.10 & -25.8 & 37.04 & 28.86 & -22.1 \\
\hline Ocean & 21.00 & 25.00 & 19.0 & 18.00 & 22.25 & 23.6 \\
\hline Total transportation & 80.40 & 69.10 & -14.1 & 55.04 & 51.11 & -7.1 \\
\hline Farm gate price ${ }^{3}$ & 275.38 & 282.59 & 2.6 & 298.43 & 300.23 & 0.6 \\
\hline Landed cost & 355.78 & 351.70 & -1.1 & 353.47 & 351.33 & -0.6 \\
\hline \multirow[t]{2}{*}{ Transport \% of landed cost } & 22.6 & 19.6 & -13.1 & 15.6 & 14.5 & -6.6 \\
\hline & \multicolumn{3}{|c|}{$\begin{array}{c}\text { Southwest } \mathrm{PI}^{1} \text { - São Luís } \\
- \text { US } \$ \mathrm{mt}-\end{array}$} & \multicolumn{3}{|c|}{$\begin{array}{c}\text { North } \mathrm{MT}^{1} \text { - Barcarena }{ }^{2} \\
\text {--US\$/mt-- }\end{array}$} \\
\hline Truck & 45.24 & 32.49 & -28.2 & 53.99 & 37.11 & -31.3 \\
\hline Barge $^{4}$ & - & - & - & 19.66 & 16.42 & -16.5 \\
\hline Ocean & 18.00 & 22.25 & 23.6 & 19.00 & 24.00 & 26.3 \\
\hline Total transportation & 63.24 & 54.74 & -13.4 & 92.65 & 77.53 & -16.3 \\
\hline Farm gate price ${ }^{3}$ & 292.96 & 302.03 & 3.1 & 275.38 & 282.59 & 2.6 \\
\hline Landed cost & 356.20 & 356.77 & 0.2 & 368.03 & 360.12 & -2.1 \\
\hline Transport $\%$ of landed cost & 17.8 & 15.3 & -13.6 & 25.2 & 21.5 & -14.5 \\
\hline
\end{tabular}

${ }^{1}$ Producing regions: $\mathrm{MT}=$ Mato Grosso, $\mathrm{PI}=$ Piauí, and MA = Maranhão.

${ }^{2}$ Export port.

${ }^{3}$ The source of the farm gate price is the Brazilian Government, Companhia Nacional de Abastecimento (CONAB).

${ }^{4}$ In Brazil, there are no public/official Barge rates. Barge rates can be up to 60 percent lower than truck rates, depending on volumes hauled and the terms of contracts signed between the barge company and shippers. The distance is in nautical miles.

Note: qtr. $=$ quarter. $\mathrm{mt}=$ metric ton

Source: University of São Paulo, Escola Superior de Agricultura "Luiz de Queiroz," Brazil (ESALQ/ USP) and USDA, Agricultural Marketing Service. 


\section{Brazil Soybean Transportation}

\section{Indicators}

Figure 2. Routes $^{1}$ and regions considered in the Brazilian soybean export transportation indicator ${ }^{2}$

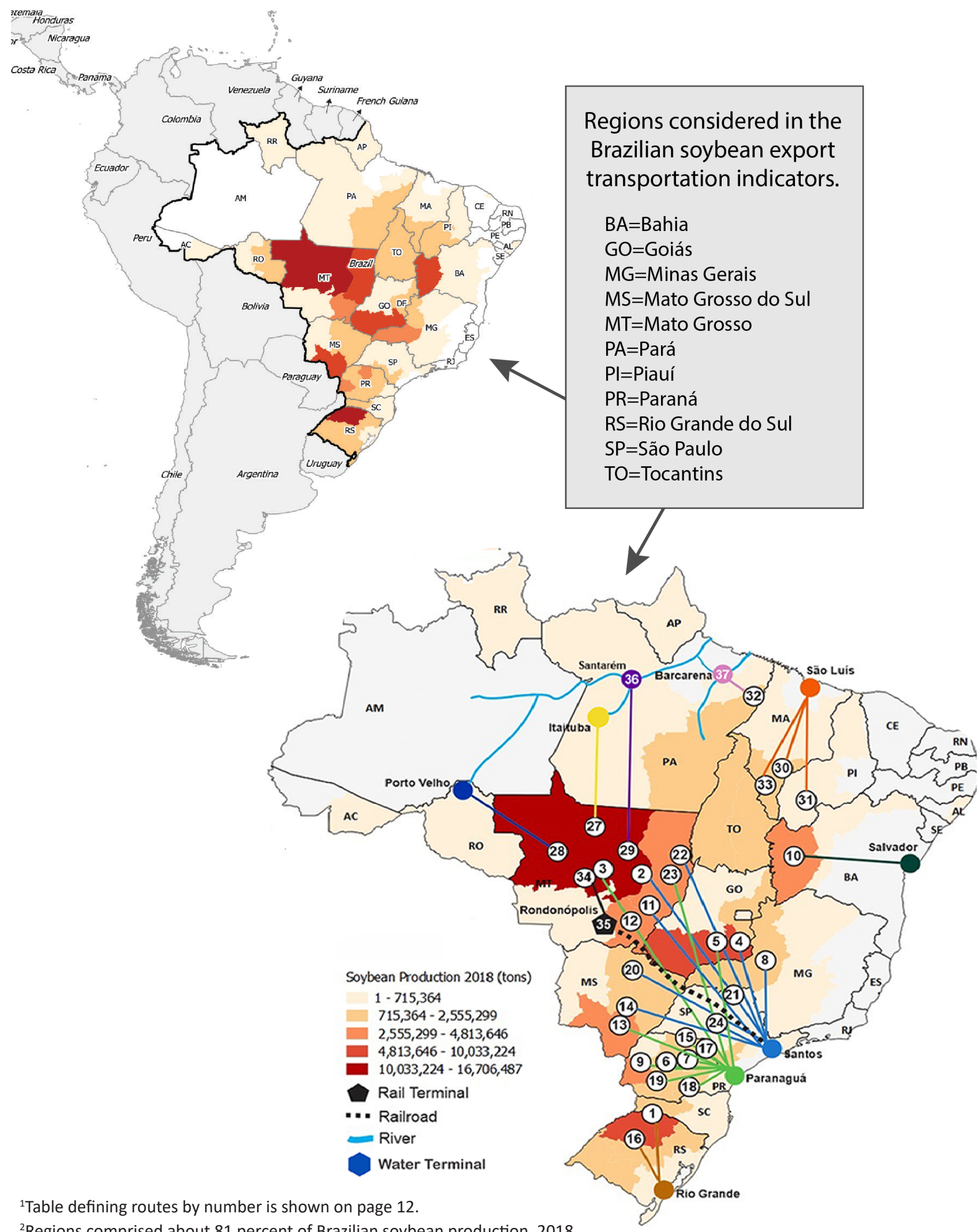

${ }^{2}$ Regions comprised about 81 percent of Brazilian soybean production, 2018.

Source: University of São Paulo, Escola Superior de Agricultura "Luiz de Queiroz," Brazil (ESALQ/ USP) and USDA, Agricultural Marketing Service. 


\section{Brazil Soybean Transportation}

Table 3. Quarterly costs of transporting Brazilian soybeans from the southern ports to Shanghai, China

\begin{tabular}{|c|c|c|c|c|c|c|c|c|c|c|}
\hline & \multicolumn{10}{|c|}{$-2020-$} \\
\hline & 1st qtr. & 2nd qtr. & 3rd qtr. & 4th qtr. & Avg. & 1st qtr. & 2nd qtr. & 3rd qtr. & 4th qtr. & Avg. \\
\hline & \multicolumn{5}{|c|}{$\begin{array}{l}\text { North } \mathrm{MT}^{1}-\text { Santos }^{2} \\
\text {-US\$ } / \mathrm{mt}-\end{array}$} & \multicolumn{5}{|c|}{$\begin{array}{c}\text { North } \mathrm{MT}^{1} \text { - Paranaguá } \\
\text {-US\$/mt- }\end{array}$} \\
\hline Truck & 68.33 & & & & 68.33 & 67.48 & & & & 67.48 \\
\hline Ocean & 35.50 & & & & 35.50 & 37.25 & & & & 37.25 \\
\hline Total transportation & 103.83 & & & & 103.83 & 104.73 & & & & 104.73 \\
\hline Farm gate price ${ }^{3}$ & 282.59 & & & & 282.59 & 282.59 & & & & 282.59 \\
\hline Landed cost & 386.43 & & & & 386.43 & 387.32 & & & & 387.32 \\
\hline \multirow[t]{2}{*}{ Transport $\%$ of landed cost } & 26.9 & & & & 26.9 & 27.0 & & & & 27.0 \\
\hline & \multicolumn{5}{|c|}{$\begin{array}{l}\text { North } \mathrm{MT}^{1} \text { - Santos } \\
\text {-US\$/mt- }\end{array}$} & \multicolumn{5}{|c|}{$\begin{array}{c}\text { Northwest RS }{ }^{1} \text { - Rio Grande } \\
\text {-US\$/mt- }\end{array}$} \\
\hline Truck & 24.79 & & & & 24.79 & 22.92 & & & & 22.92 \\
\hline Rail $^{4}$ & 37.73 & & & & 37.73 & - & & & & - \\
\hline Ocean & 35.50 & & & & 35.50 & 37.00 & & & & 37.00 \\
\hline Total transportation & 98.02 & & & & 98.02 & 59.92 & & & & 59.92 \\
\hline Farm gate price ${ }^{3}$ & 282.59 & & & & 282.59 & 300.04 & & & & 300.04 \\
\hline Landed cost & 380.61 & & & & 380.61 & 359.97 & & & & 359.97 \\
\hline Transport $\%$ of landed cost & 25.8 & & & & 25.8 & 16.6 & & & & 16.6 \\
\hline
\end{tabular}

${ }^{1}$ Producing regions: RS = Rio Grande Do Sul, MT= Mato Grosso, and PR = Paraná.

${ }^{2}$ Export port.

${ }^{3}$ The source of the farm gate price is the Brazilian Government, Companhia Nacional de Abastecimento (CONAB).

${ }^{4}$ In, Brazil, there are no public/official rail tariff rates. Rail rates can be up to 30 percent lower than truck rates, depending on volumes hauled and the terms of contracts signed between the railroad company and shippers.

Note: qtr. = quarter. $\mathrm{mt}=$ metric ton. Avg. = average.

Source: University of São Paulo, Escola Superior de Agricultura “Luiz de Queiroz," Brazil (ESALQ/ USP) and USDA, Agricultural Marketing Service. 


\section{Brazil Soybean Transportation}

Table 4. Quarterly costs of transporting Brazilian soybeans from the southern ports to Hamburg, Germany

\begin{tabular}{|c|c|c|c|c|c|c|c|c|c|c|}
\hline & \multicolumn{10}{|c|}{$-2020-$} \\
\hline & 1st qtr. & 2nd qtr. & 3rd qtr. & 4th qtr. & Avg. & 1st qtr. & 2nd qtr. & 3rd qtr. & 4th qtr. & Avg. \\
\hline & \multicolumn{5}{|c|}{$\begin{array}{l}\text { North } \mathrm{MT}^{1} \text { - Santos } \\
\text {-US } \$ / \mathrm{mt}-\end{array}$} & \multicolumn{5}{|c|}{$\begin{array}{c}\text { North } \mathrm{MT}^{1} \text { - Paranaguá } \\
\text {-US\$/mt- }\end{array}$} \\
\hline Truck & 68.33 & & & & 68.33 & 67.48 & & & & 67.48 \\
\hline Ocean & 29.25 & & & & 29.25 & 30.00 & & & & 30.00 \\
\hline Total transportation & 97.58 & & & & 97.58 & 97.48 & & & & 97.48 \\
\hline Farm gate price ${ }^{3}$ & 282.59 & & & & 282.59 & 282.59 & & & & 282.59 \\
\hline Landed cost & 380.18 & & & & 380.18 & 380.07 & & & & 380.07 \\
\hline \multirow[t]{2}{*}{ Transport \% of landed cost } & 25.7 & & & & 25.7 & 25.6 & & & & 25.6 \\
\hline & \multicolumn{5}{|c|}{$\begin{array}{l}\text { North } \mathrm{MT}^{1} \text { - Santos } \\
\text {-US\$/mt- }\end{array}$} & \multicolumn{5}{|c|}{$\begin{array}{c}\text { Northwest RS }{ }^{1} \text { - Rio Grande } \\
\text {-US\$/mt- }\end{array}$} \\
\hline Truck & 24.79 & & & & 24.79 & 22.92 & & & & 22.92 \\
\hline Rail $^{4}$ & 37.73 & & & & 37.73 & - & & & & - \\
\hline Ocean & 29.25 & & & & 29.25 & 29.50 & & & & 29.50 \\
\hline Total transportation & 91.77 & & & & 91.77 & 52.42 & & & & 52.42 \\
\hline Farm gate price ${ }^{3}$ & 282.59 & & & & 282.59 & 300.04 & & & & 300.04 \\
\hline Landed cost & 374.36 & & & & 374.36 & 352.47 & & & & 352.47 \\
\hline Transport \% of landed cost & 24.5 & & & & 24.5 & 14.9 & & & & 14.9 \\
\hline
\end{tabular}

${ }^{1}$ Producing regions: $\mathrm{RS}=$ Rio Grande Do Sul, MT= Mato Grosso, and PR = Paraná.

${ }^{2}$ Export port.

${ }^{3}$ The source of the farm gate price is the Brazilian Government, Companhia Nacional de Abastecimento (CONAB).

${ }^{4} \mathrm{In}$, Brazil, there are no public/official rail tariff rates. Rail rates can be up to 30 percent lower than truck rates, depending on volumes hauled and the terms of contracts signed between the railroad company and shippers.

Note: qtr. $=$ quarter $. \mathrm{mt}=$ metric ton. Avg. $=$ average .

Source: University of São Paulo, Escola Superior de Agricultura "Luiz de Queiroz," Brazil (ESALQ/ USP) and USDA, Agricultural Marketing Service. 


\section{Brazil Soybean Transportation}

Table 5. Quarterly costs of transporting Brazilian soybeans from the northern and northeastern ports to Shanghai, China

\begin{tabular}{|c|c|c|c|c|c|c|c|c|c|c|}
\hline & \multicolumn{10}{|c|}{$-2020-$} \\
\hline & 1st qtr. & 2nd qtr. & 3rd qtr. & 4th qtr. & Avg. & 1st qtr. & 2nd qtr. & 3rd qtr. & 4th qtr. & Avg. \\
\hline & \multicolumn{5}{|c|}{$\begin{array}{c}\text { North } \mathrm{MT}^{1} \text { - Santarém } \\
\text {-US\$/mt- }\end{array}$} & \multicolumn{5}{|c|}{$\begin{array}{c}\text { South } \mathrm{MA}^{1} \text { - São Luís } \\
\text {-US\$/mt- }\end{array}$} \\
\hline Truck & 44.10 & & & & 44.10 & 28.86 & & & & 28.86 \\
\hline Ocean & 36.50 & & & & 36.50 & 36.75 & & & & 36.75 \\
\hline Total transportation & 80.60 & & & & 80.60 & 65.61 & & & & 65.61 \\
\hline Farm gate price ${ }^{3}$ & 282.59 & & & & 282.59 & 300.23 & & & & 300.23 \\
\hline Landed cost & 363.20 & & & & 363.20 & 365.83 & & & & 365.83 \\
\hline \multirow[t]{2}{*}{ Transport $\%$ of landed cost } & 22.2 & & & & 22.2 & 17.9 & & & & 17.9 \\
\hline & \multicolumn{5}{|c|}{$\begin{array}{c}\text { Southwest } \mathrm{PI}^{1} \text { - São Luís } \\
\text {-US\$/mt- }\end{array}$} & \multicolumn{5}{|c|}{$\begin{array}{c}\text { North } \mathrm{MT}^{1} \text { - Barcarena } \\
\text {--US\$/mt- }\end{array}$} \\
\hline Truck & 32.49 & & & & 32.49 & 37.11 & & & & 37.11 \\
\hline Barge $^{4}$ & - & & & & - & 16.42 & & & & 16.42 \\
\hline Ocean & 36.75 & & & & 36.75 & 38.50 & & & & 38.50 \\
\hline Total transportation & 69.24 & & & & 69.24 & 92.03 & & & & 92.03 \\
\hline Farm gate price ${ }^{3}$ & 302.03 & & & & 302.03 & 282.59 & & & & 282.59 \\
\hline Landed cost & 371.27 & & & & 371.27 & 374.62 & & & & 374.62 \\
\hline Transport \% of landed cost & 18.6 & & & & 18.6 & 24.6 & & & & 24.6 \\
\hline
\end{tabular}

${ }^{1}$ Producing regions: $\mathrm{MT}=$ Mato Grosso, $\mathrm{PI}=$ Piauí, and MA = Maranhão.

${ }^{2}$ Export port.

${ }^{3}$ The source of the farm gate price is the Brazilian Government, Companhia Nacional de Abastecimento (CONAB).

${ }^{4}$ In Brazil, there are no public/official Barge rates. Barge rates can be up to 60 percent lower than truck rates, depending on volumes hauled and the terms of contracts signed between the barge company and shippers. The distance is in nautical miles.

Note: qtr. = quarter. $\mathrm{mt}=$ metric ton. Avg. = average.

Source: University of São Paulo, Escola Superior de Agricultura “Luiz de Queiroz," Brazil (ESALQ/ USP) and USDA, Agricultural Marketing Service. 


\section{Brazil Soybean Transportation}

Table 6. Quarterly costs of transporting Brazilian soybeans from the northern and northeastern ports to Hamburg, Germany

\begin{tabular}{|c|c|c|c|c|c|c|c|c|c|c|}
\hline & \multicolumn{10}{|c|}{$-2020-$} \\
\hline & 1st qtr. & 2nd qtr. & 3rd qtr. & 4th qtr. & Avg. & 1st qtr. & 2nd qtr. & 3rd qtr. & 4th qtr. & Avg. \\
\hline & \multicolumn{5}{|c|}{$\begin{array}{c}\text { North } \mathrm{MT}^{1} \text { - Santarém }{ }^{2} \\
\text {-US\$/mt- }\end{array}$} & \multicolumn{5}{|c|}{$\begin{array}{c}\text { South } \mathrm{MA}^{1} \text { - São Luís } \\
-\mathrm{US} \$ \mathrm{mt}-\end{array}$} \\
\hline Truck & 44.10 & & & & 44.10 & 28.86 & & & & 28.86 \\
\hline Ocean & 25.00 & & & & 25.00 & 22.25 & & & & 22.25 \\
\hline Total transportation & 69.10 & & & & 69.10 & 51.11 & & & & 51.11 \\
\hline Farm gate price ${ }^{3}$ & 282.59 & & & & 282.59 & 300.23 & & & & 300.23 \\
\hline Landed cost & 351.70 & & & & 351.70 & 351.33 & & & & 351.33 \\
\hline \multirow[t]{2}{*}{ Transport \% of landed cost } & 19.6 & & & & 19.6 & 14.5 & & & & 14.5 \\
\hline & \multicolumn{5}{|c|}{$\begin{array}{c}\text { Southwest } \text { PI }^{1} \text { - São Luís } \\
\text {-US\$/mt- }\end{array}$} & \multicolumn{5}{|c|}{$\begin{array}{c}\text { North } \mathrm{MT}^{1} \text { - Barcarena } \\
\text {--US\$/mt- }\end{array}$} \\
\hline Truck & 32.49 & & & & 32.49 & 37.11 & & & & 37.11 \\
\hline Barge $^{4}$ & - & & & & - & 16.42 & & & & 16.42 \\
\hline Ocean & 22.25 & & & & 22.25 & 24.00 & & & & 24.00 \\
\hline Total transportation & 54.74 & & & & 54.74 & 77.53 & & & & 77.53 \\
\hline Farm gate price ${ }^{3}$ & 302.03 & & & & 302.03 & 282.59 & & & & 282.59 \\
\hline Landed cost & 356.77 & & & & 356.77 & 360.12 & & & & 360.12 \\
\hline Transport \% of landed cost & 15.3 & & & & 15.3 & 21.5 & & & & 21.5 \\
\hline
\end{tabular}

${ }^{1}$ Producing regions: $\mathrm{MT}=$ Mato Grosso, $\mathrm{PI}=$ Piauí, and $\mathrm{MA}=$ Maranhão.

${ }^{2}$ Export port.

${ }^{3}$ The source of the farm gate price is the Brazilian Government, Companhia Nacional de Abastecimento (CONAB).

${ }^{4}$ In Brazil, there are no public/official Barge rates. Barge rates can be up to 60 percent lower than truck rates, depending on volumes hauled and the terms of contracts signed between the barge company and shippers. The distance is in nautical miles.

Note: qtr. = quarter. $\mathrm{mt}=$ metric ton. Avg. = average.

Source: University of São Paulo, Escola Superior de Agricultura "Luiz de Queiroz," Brazil (ESALQ/ USP) and USDA, Agricultural Marketing Service. 


\section{Brazil Soybean Transportation}

Table 7. Quarterly truck rates for selected Brazilian soybean export transportation routes, 2020

\begin{tabular}{|c|c|c|c|c|c|c|c|c|c|}
\hline \multirow{2}{*}{$\begin{array}{c}\text { Route } \\
\#\end{array}$} & \multirow{2}{*}{$\begin{array}{l}\text { Origin }^{1} \\
\text { (reference city) }\end{array}$} & \multirow{2}{*}{ Destination } & \multirow{2}{*}{$\begin{array}{l}\text { Distance } \\
\text { (miles) }^{2}\end{array}$} & \multirow{2}{*}{$\begin{array}{l}\text { Share } \\
(\%)^{3}\end{array}$} & \multicolumn{5}{|c|}{ Freight price (US\$\$/mt/100 miles) ${ }^{4}$} \\
\hline & & & & & 1st qtr. & 2nd qtr. & 3rd qtr. & 4th qtr. & Avg. \\
\hline 1 & Northwest RS 5 (Cruz Alta) & Rio Grande & 288 & 11.3 & 7.96 & & & & 7.96 \\
\hline 2 & North MT (Sorriso) & Santos & 1,190 & 3.0 & 5.74 & & & & 5.74 \\
\hline 3 & North MT (Sorriso) & Paranaguá & 1,262 & 2.9 & 5.35 & & & & 5.35 \\
\hline 4 & South GO (Rio Verde) & Santos & 587 & 4.8 & 5.54 & & & & 5.54 \\
\hline 5 & South GO (Rio Verde) & Paranaguá & 726 & 3.9 & 5.60 & & & & 5.60 \\
\hline 6 & North Central PR (Londrina) & Paranaguá & 268 & 3.2 & 8.00 & & & & 8.00 \\
\hline 7 & Western Central PR (Mamborê) & Paranaguá & 311 & 2.4 & 7.22 & & & & 7.22 \\
\hline 8 & Triangle MG (Uberaba) & Santos & 339 & 3.0 & 7.66 & & & & 7.66 \\
\hline 9 & West PR (Assis Chateaubriand) & Paranaguá & 377 & 2.3 & 6.71 & & & & 6.71 \\
\hline 10 & West Extreme BA (São Desidério) & Salvador & 535 & 6.6 & 5.97 & & & & 5.97 \\
\hline 11 & Southeast MT (Primavera do Leste) & Santos & 901 & 2.4 & 5.26 & & & & 5.26 \\
\hline 12 & Southeast MT (Primavera do Leste) & Paranaguá & 975 & 2.2 & 5.05 & & & & 5.05 \\
\hline 13 & Southwest MS (Maracaju) & Paranaguá & 612 & 3.5 & 5.99 & & & & 5.99 \\
\hline 14 & Southwest MS (Maracaju) & Santos & 652 & 3.2 & 5.82 & & & & 5.82 \\
\hline 15 & West PR (Assis Chateaubriand) & Santos & 550 & 1.6 & 5.94 & & & & 5.94 \\
\hline 16 & East GO (Cristalina) & Santos & 585 & 1.9 & 6.35 & & & & 6.35 \\
\hline 17 & North PR (Cornélio Procópio) & Paranaguá & 306 & 1.8 & 6.46 & & & & 6.46 \\
\hline 18 & Eastern Central PR (Castro) & Paranaguá & 130 & 2.0 & 10.54 & & & & 10.54 \\
\hline 19 & South Central PR (Guarapuava) & Paranaguá & 204 & 2.3 & 9.63 & & & & 9.63 \\
\hline 20 & North Central MS (São Gabriel do Oeste) & Santos & 720 & 2.4 & 5.06 & & & & 5.06 \\
\hline 21 & Ribeirão Preto SP (Guairá) & Santos & 314 & 0.0 & 6.40 & & & & 6.40 \\
\hline 22 & Northeast MT (Canarana) & Santos & 950 & 3.3 & 5.55 & & & & 5.55 \\
\hline 23 & East MS (Chapadão do Sul) & Santos & 607 & 0.0 & 5.08 & & & & 5.08 \\
\hline 24 & Northeast MT (Canarana) & Paranaguá & 1,075 & 2.9 & 5.34 & & & & 5.34 \\
\hline
\end{tabular}

${ }^{1}$ Although each origin region comprises several cities, the main city is considered as a reference to establish the freight price; na = not available ${ }^{2}$ Distance from the main city of the considered region to the mentioned ports.

${ }^{3}$ Share is measured as a percentage of total production.

${ }^{4}$ Average monthly exchange rate from "Banco Central do Brasil" was used to convert Brazilian reais to the U.S. dollars.

${ }^{5} \mathrm{RS}=$ Rio Grande do Sul, MT=Mato Grosso, GO=Goiás, PR=Paraná, MG=Minas Gerais, BA=Bahia, MS=Mato Grosso do Sul, SP=São Paulo, PI=Piauí, $\mathrm{MA}=$ Maranhão, $\mathrm{PA}=$ Pará, and TO=Tocantins.

${ }^{6}$ In Brazil, there are no public/official rail tariff rates. Rail rates can be up to 30 percent lower than truck rates, depending on the volumes hauled and the terms of contracts signed between the railroad company and shippers.

${ }^{7}$ In Brazil, there are no public/official Barge rates. Barge rates can be up to 60 percent lower than truck rates, depending on the volumes hauled and the terms of contracts signed between the barge company and shippers. The distance is in nautical miles.

Note: qtr. = quarter. $\mathrm{mt}=$ metric ton. Avg. = average.

Source: University of São Paulo, Escola Superior de Agricultura "Luiz de Queiroz," Brazil (ESALQ/ USP) and USDA, Agricultural Marketing Service. 


\section{Brazil Soybean Transportation}

Table 7. Quarterly truck rates for selected Brazilian soybean export transportation routes, 2020

\begin{tabular}{|c|c|c|c|c|c|c|c|c|c|}
\hline \multirow{2}{*}{$\begin{array}{c}\text { Route } \\
\#\end{array}$} & \multirow{2}{*}{$\begin{array}{c}\text { Origin }^{1} \\
\text { (reference city) }\end{array}$} & \multirow{2}{*}{ Destination } & \multirow{2}{*}{$\begin{array}{l}\text { Distance } \\
\text { (miles) }^{2}\end{array}$} & \multirow{2}{*}{$\begin{array}{c}\text { Share } \\
(\%)^{3}\end{array}$} & \multicolumn{5}{|c|}{ Freight price (US\$/mt/100 miles) $)^{4}$} \\
\hline & & & & & 1st qtr. & 2nd qtr. & 3rd qtr. & 4th qtr. & Avg. \\
\hline 25 & Western Central RS (Tupanciretã) & Rio Grande & 273 & 2.5 & 7.10 & & & & 7.10 \\
\hline 26 & Southwest PR(Chopinzinho) & Paranaguá & 291 & 1.7 & 7.57 & & & & 7.57 \\
\hline 27 & North MT (Sorriso) & Itaituba & 672 & 5.4 & 5.52 & & & & 5.52 \\
\hline 28 & North MT (Sorriso) & Porto Velho & 632 & 5.7 & 5.22 & & & & 5.22 \\
\hline 29 & North MT (Sorriso) & Santarém & 876 & 4.1 & 5.03 & & & & 5.03 \\
\hline 30 & South MA (Balsas) & São Luís & 482 & 2.1 & 5.99 & & & & 5.99 \\
\hline 31 & Southwest PI (Bom Jesus) & São Luís & 606 & 2.6 & 5.36 & & & & 5.36 \\
\hline 32 & Southeast PA (Paragominas) & Barcarena & 249 & 1.5 & 7.17 & & & & 7.17 \\
\hline 33 & East TO (Campos Lindos) & São Luís & 842 & 1.6 & 5.04 & & & & 5.04 \\
\hline & Weighted average & & 587 & 100.0 & 6.33 & & & & 6.33 \\
\hline 34 & North MT (Sorriso) & $\begin{array}{l}\text { Rondonópolis } \\
\text { (Rail terminal) }\end{array}$ & 382 & & 6.49 & & & & 6.49 \\
\hline 35 & Rondonópolis MT (Rail terminal) ${ }^{6}$ & Santos & 1,019 & & 3.70 & & & & 3.70 \\
\hline 36 & Itaituba PA (Barge terminal)7 & Santarém & 224 & & 4.73 & & & & 4.73 \\
\hline 37 & Itaituba PA (Barge terminal)7 & Barcarena & 738 & & 2.23 & & & & 2.23 \\
\hline
\end{tabular}

${ }^{1}$ Although each origin region comprises several cities, the main city is considered as a reference to establish the freight price; na = not available ${ }^{2}$ Distance from the main city of the considered region to the mentioned ports.

${ }^{3}$ Share is measured as a percentage of total production.

${ }^{4}$ Average monthly exchange rate from "Banco Central do Brasil" was used to convert Brazilian reais to the U.S. dollars.

${ }^{5} \mathrm{RS}=$ Rio Grande do Sul, MT=Mato Grosso, GO=Goiás, PR=Paraná, MG=Minas Gerais, BA=Bahia, MS=Mato Grosso do Sul, SP=São Paulo, PI=Piauí, MA=Maranhão, PA=Pará, and TO=Tocantins.

${ }^{6}$ In Brazil, there are no public/official rail tariff rates. Rail rates can be up to 30 percent lower than truck rates, depending on the volumes hauled and the terms of contracts signed between the railroad company and shippers.

${ }^{7}$ In Brazil, there are no public/official Barge rates. Barge rates can be up to 60 percent lower than truck rates, depending on the volumes hauled and the terms of contracts signed between the barge company and shippers. The distance is in nautical miles.

Note: qtr. = quarter. $\mathrm{mt}=$ metric ton. Avg. = average.

Source: University of São Paulo, Escola Superior de Agricultura "Luiz de Queiroz," Brazil (ESALQ/ USP) and USDA, Agricultural Marketing Service. 


\section{Brazil Soybean Transportation}

Table 8. Monthly Brazilian soybean export truck transportation cost index

\begin{tabular}{|c|c|c|c|c|c|c|c|}
\hline Month & $\begin{array}{c}\text { Freight price } \\
\text { (US\$/mt/100 miles) }\end{array}$ & $\begin{array}{l}\text { Index variation (\%) } \\
\text { (Base: prior month) }\end{array}$ & $\begin{array}{c}\text { Index value } \\
\text { (Base: Jan-05=100) }\end{array}$ & Month & $\begin{array}{c}\text { Freight price } \\
\text { (US\$/mt/100 miles) }\end{array}$ & $\begin{array}{l}\text { Index variation (\%) } \\
\text { (Base: prior month) }\end{array}$ & $\begin{array}{c}\text { Index value } \\
\text { (Base: Jan-05=100) }\end{array}$ \\
\hline Jan-13 & 10.11 & 3.9 & 174.31 & Jan-17 & 7.32 & 33.8 & 126.20 \\
\hline Feb-13 & 10.79 & 6.7 & 185.96 & Feb-17 & 9.85 & 34.6 & 169.85 \\
\hline Mar-13 & 11.14 & 3.3 & 192.04 & Mar-17 & 10.38 & 5.3 & 178.90 \\
\hline Apr-13 & 10.95 & -1.7 & 188.71 & Apr-17 & 9.52 & -8.3 & 164.05 \\
\hline May-13 & 10.40 & -5.0 & 179.31 & May-17 & 8.75 & -8.0 & 150.90 \\
\hline Jun-13 & 9.49 & -8.8 & 163.61 & Jun-17 & 8.18 & -6.5 & 141.04 \\
\hline Jul-13 & 9.65 & 1.7 & 166.41 & Jul-17 & 8.74 & 6.8 & 150.66 \\
\hline Aug-13 & 9.80 & 1.5 & 168.95 & Aug-17 & 9.85 & 12.7 & 169.76 \\
\hline Sep-13 & 10.21 & 4.2 & 176.02 & Sep-17 & 8.97 & -9.0 & 154.55 \\
\hline Oct-13 & 10.17 & -0.4 & 175.28 & Oct-17 & 8.64 & -3.6 & 148.93 \\
\hline Nov-13 & 9.29 & -8.6 & 160.18 & Nov-17 & 8.36 & -3.2 & 144.11 \\
\hline Dec-13 & 8.91 & -4.1 & 153.63 & Dec-17 & 7.23 & -13.5 & 124.63 \\
\hline Jan-14 & 8.86 & -0.6 & 152.73 & Jan-18 & 7.59 & 5.0 & 130.90 \\
\hline Feb-14 & 10.34 & 16.7 & 178.24 & Feb-18 & 8.65 & 13.9 & 149.04 \\
\hline Mar-14 & 11.61 & 12.3 & 200.13 & Mar-18 & 10.59 & 22.5 & 182.61 \\
\hline Apr-14 & 11.35 & -2.2 & 195.65 & Apr-18 & 9.78 & -7.7 & 168.59 \\
\hline May-14 & 10.90 & -4.0 & 187.89 & May-18 & 8.96 & -8.4 & 154.45 \\
\hline Jun-14 & 10.34 & -5.1 & 178.24 & Jun-18 & 8.89 & -0.8 & 153.24 \\
\hline Jul-14 & 10.16 & -1.7 & 175.21 & Jul-18 & 8.97 & 0.9 & 154.58 \\
\hline Aug-14 & 10.10 & -0.6 & 174.08 & Aug-18 & 8.24 & -8.1 & 142.00 \\
\hline Sep-14 & 9.66 & -4.3 & 166.54 & Sep-18 & 7.24 & -12.1 & 124.78 \\
\hline Oct-14 & 8.77 & -9.3 & 151.13 & Oct-18 & 7.69 & 6.2 & 132.55 \\
\hline Nov-14 & 8.36 & -4.6 & 144.16 & Nov-18 & 7.51 & -2.3 & 129.44 \\
\hline Dec-14 & 7.96 & -4.9 & 137.15 & Dec-18 & 7.19 & -4.3 & 123.87 \\
\hline Jan-15 & 8.01 & 0.7 & 138.15 & Jan-19 & 7.72 & 7.5 & 133.13 \\
\hline Feb-15 & 8.02 & 0.1 & 138.29 & Feb-19 & 8.19 & 6.0 & 141.15 \\
\hline Mar-15 & 8.32 & 3.7 & 143.44 & Mar-19 & 7.34 & -10.3 & 126.61 \\
\hline Apr-15 & 9.00 & 8.2 & 155.13 & Apr-19 & 7.16 & -2.6 & 123.35 \\
\hline May-15 & 8.39 & -6.8 & 144.58 & May-19 & 6.73 & -5.9 & 116.02 \\
\hline Jun-15 & 8.01 & -4.5 & 138.12 & Jun-19 & 6.94 & 3.1 & 119.56 \\
\hline Jul-15 & 7.56 & -5.7 & 130.25 & Jul-19 & 8.33 & 20.1 & 143.60 \\
\hline Aug-15 & 7.38 & -2.4 & 127.15 & Aug-19 & 7.85 & -5.8 & 135.23 \\
\hline Sep-15 & 6.60 & -10.5 & 113.78 & Sep-19 & 7.09 & -9.7 & 122.17 \\
\hline Oct-15 & 6.70 & 1.5 & 115.43 & Oct-19 & 6.57 & -7.4 & 113.19 \\
\hline Nov-15 & 7.08 & 5.8 & 122.08 & Nov-19 & 6.41 & -2.3 & 110.54 \\
\hline Dec-15 & 6.76 & -4.5 & 116.56 & Dec-19 & 5.93 & -7.5 & 102.21 \\
\hline Jan-16 & 6.42 & -5.1 & 110.63 & Jan-20 & 6.03 & 1.7 & 103.90 \\
\hline Feb-16 & 6.73 & 4.8 & 115.98 & Feb-20 & 6.76 & 12.2 & 116.52 \\
\hline Mar-16 & 7.79 & 15.8 & 134.33 & Mar-20 & 6.20 & -8.2 & 106.95 \\
\hline Apr-16 & 8.30 & 6.5 & 143.05 & & & & \\
\hline May-16 & 7.28 & -12.3 & 125.43 & & & & \\
\hline Jun-16 & 7.16 & -1.5 & 123.51 & & & & \\
\hline Jul-16 & 7.46 & 4.2 & 128.64 & & & & \\
\hline Aug-16 & 7.33 & -1.7 & 126.41 & & & & \\
\hline Sep-16 & 6.35 & -13.3 & 109.53 & & & & \\
\hline Oct-16 & 5.88 & -7.5 & 101.35 & & & & \\
\hline Nov-16 & 5.00 & -14.9 & 86.21 & & & & \\
\hline Dec-16 & 5.47 & 9.4 & 94.32 & & & & \\
\hline
\end{tabular}

*Weighted average.

Source: University of São Paulo, Escola Superior de Agricultura "Luiz de

Queiroz," Brazil (ESALQ/ USP) and USDA, Agricultural Marketing Service. 


\section{Brazil Soybean Transportation}

Figure 3. Truck rates for selected southern Brazilian soybean export transportation routes, 2017-20

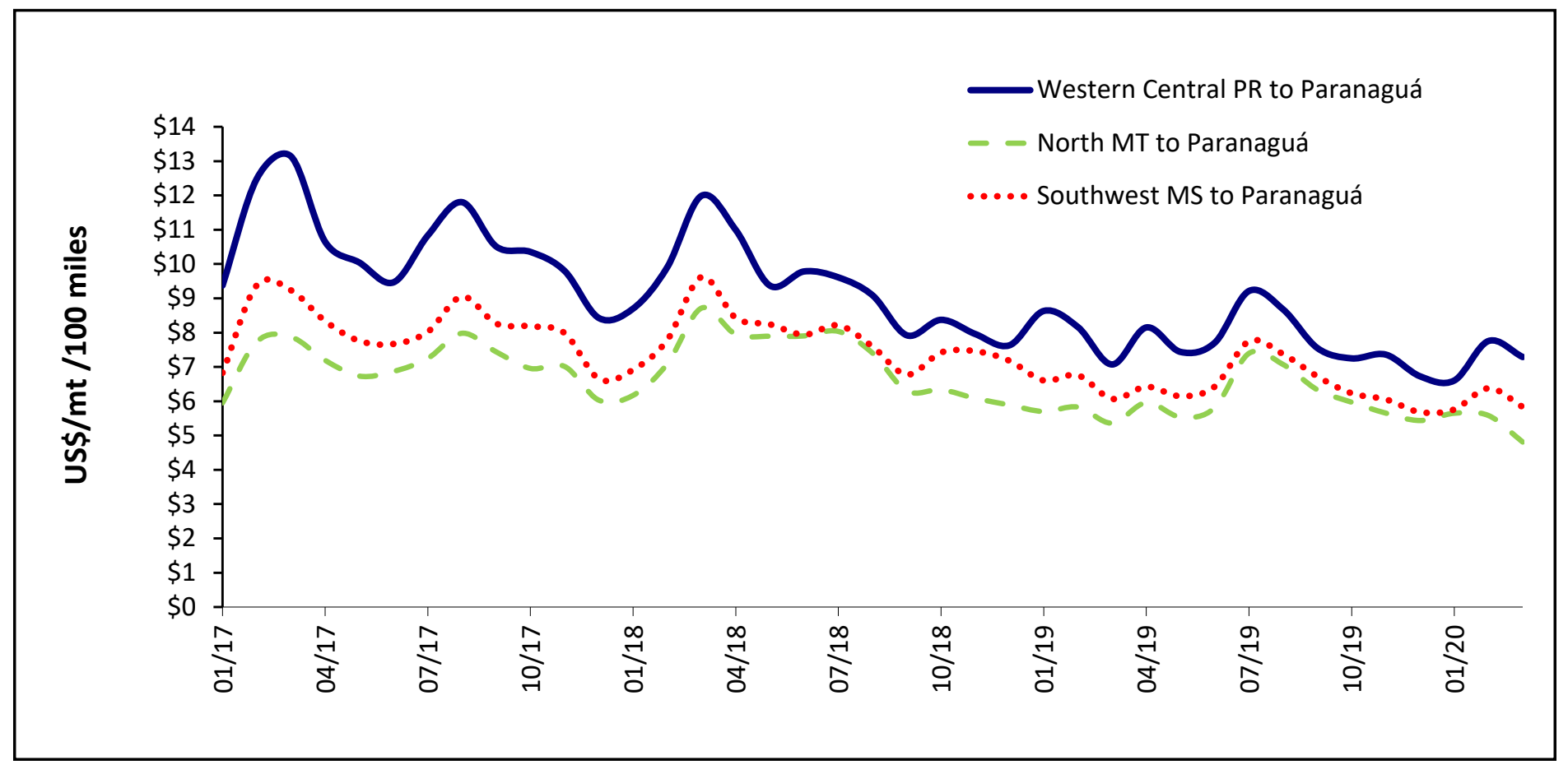

Note: $\mathrm{mt}=$ metric ton .

Source: University of São Paulo, Escola Superior de Agricultura "Luiz de Queiroz," Brazil (ESALQ/ USP) and USDA, Agricultural Marketing Service.

Figure 4. Truck rates for selected north, south, and northeastern Brazilian soybean export transportation routes, 2017-20

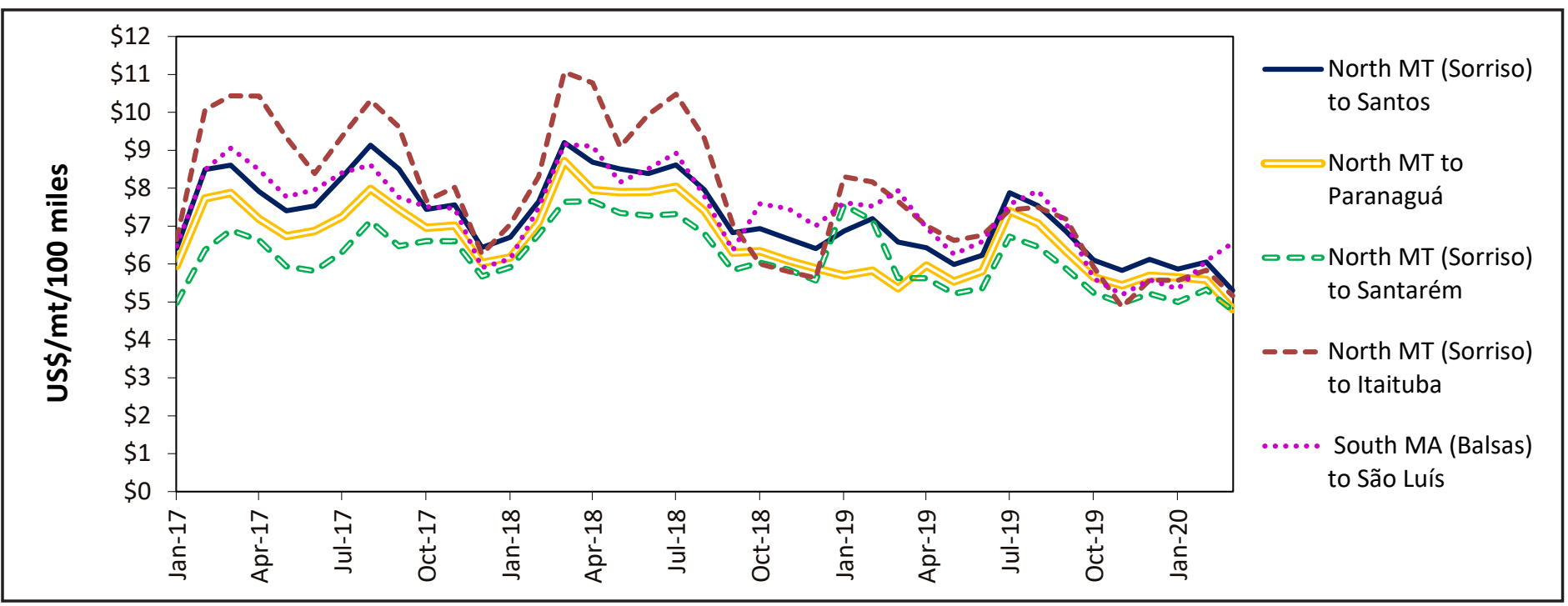

Note: $\mathrm{mt}=$ metric ton.

Source: University of São Paulo, Escola Superior de Agricultura “Luiz de Queiroz," Brazil (ESALQ/ USP) and USDA, Agricultural Marketing Service. 


\section{Brazil Soybean Transportation}

Figure 5. Brazilian soybean export truck transportation weighted average prices, 2017-20

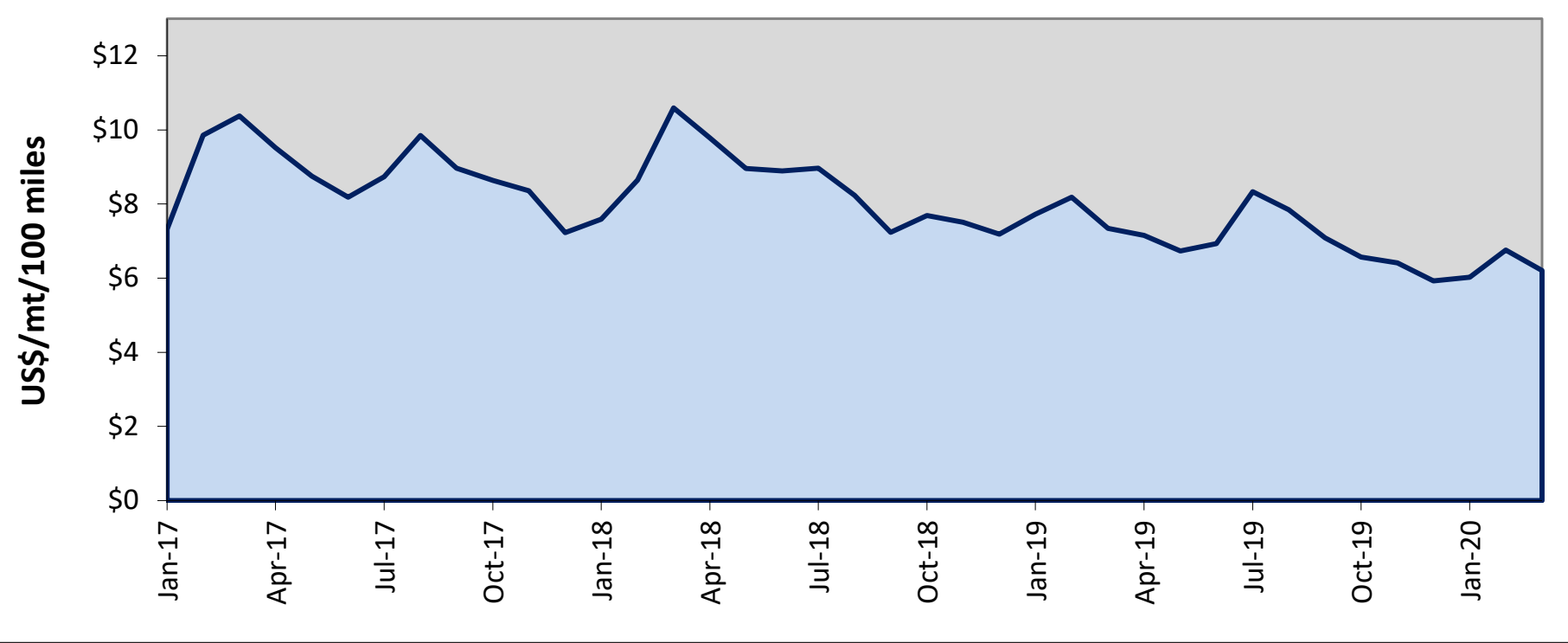

Note: $\mathrm{mt}=$ metric ton.

Source: University of São Paulo, Escola Superior de Agricultura “Luiz de Queiroz," Brazil (ESALQ/ USP) and USDA, Agricultural Marketing Service. 


\section{Brazil Soybean Transportation}

Table 9. Quarterly ocean freight rates for shipping soybeans from selected Brazilian ports to Germany and China (US\$/metric ton)*

\begin{tabular}{|c|c|c|c|c|c|}
\hline Port & Destination & 1st qtr. 2015 & 2nd qtr. 2015 & 3rd qtr. 2015 & 4th qtr. 2015 \\
\hline Santos & Germany (Hamburg) & 22.00 & 21.00 & 19.00 & 17.00 \\
\hline Paranaguá & Germany (Hamburg) & 22.00 & 21.00 & 19.00 & 17.00 \\
\hline Rio Grande & Germany (Hamburg) & 22.00 & 21.00 & 19.00 & 17.00 \\
\hline Santarém & Germany (Hamburg) & 20.00 & 14.50 & 13.50 & 20.00 \\
\hline São Luís & Germany (Hamburg) & 20.00 & 18.25 & 16.38 & 20.50 \\
\hline Barcarena & Germany (Hamburg) & 20.00 & 16.00 & 15.20 & 21.00 \\
\hline Santos & China (Shanghai) & 29.50 & 22.50 & 23.25 & 20.00 \\
\hline Paranagua & China (Shanghai) & 31.50 & 23.50 & 24.18 & 20.50 \\
\hline Rio Grande & China (Shanghai) & 29.50 & 25.00 & 25.75 & 21.00 \\
\hline Santarém & China (Shanghai) & 32.00 & 25.00 & 25.75 & 23.50 \\
\hline São Luís & China (Shanghai) & 32.00 & 25.00 & 25.75 & 23.50 \\
\hline Barcarena & China (Shanghai) & 32.00 & 25.00 & 25.75 & 23.50 \\
\hline Port & Destination & 1st qtr. 2016 & 2nd qtr. 2016 & 3rd qtr .2016 & 4th qtr. 2016 \\
\hline Santos & Germany (Hamburg) & 16.00 & 17.00 & 16.50 & 23.00 \\
\hline Paranaguá & Germany (Hamburg) & 16.00 & 17.00 & 16.50 & 24.00 \\
\hline Rio Grande & Germany (Hamburg) & 16.00 & 17.00 & 16.50 & 23.00 \\
\hline Santarém & Germany (Hamburg) & 11.03 & 14.13 & 15.00 & 19.80 \\
\hline São Luís & Germany (Hamburg) & 8.25 & 11.00 & 11.80 & 15.80 \\
\hline Barcarena & Germany (Hamburg) & 9.60 & 12.45 & 13.20 & 17.35 \\
\hline Santos & China (Shanghai) & 17.50 & 16.50 & 12.50 & 20.00 \\
\hline Paranagua & China (Shanghai) & 18.00 & 18.50 & 14.50 & 21.50 \\
\hline Rio Grande & China (Shanghai) & 18.50 & 17.00 & 13.00 & 20.50 \\
\hline Santarém & China (Shanghai) & 22.00 & 21.00 & 19.40 & 23.75 \\
\hline São Luís & China (Shanghai) & 20.00 & 18.40 & 17.50 & 22.00 \\
\hline Barcarena & China (Shanghai) & 22.50 & 21.50 & 20.00 & 23.75 \\
\hline Port & Destination & 1st qtr. 2017 & 2nd qtr. 2017 & 3rd qtr. 2017 & 4th qtr. 2017 \\
\hline Santos & Germany (Hamburg) & 21.00 & 24.00 & 26.00 & 27.00 \\
\hline Paranaguá & Germany (Hamburg) & 22.00 & 25.00 & 27.00 & 28.00 \\
\hline Rio Grande & Germany (Hamburg) & 22.00 & 25.00 & 27.00 & 28.00 \\
\hline Santarém & Germany (Hamburg) & 21.00 & 23.60 & 25.00 & 26.00 \\
\hline São Luís & Germany (Hamburg) & 17.60 & 20.00 & 21.20 & 22.00 \\
\hline Barcarena & Germany (Hamburg) & 18.00 & 20.60 & 21.80 & 22.70 \\
\hline Santos & China (Shanghai) & 18.50 & 29.00 & 30.00 & 30.00 \\
\hline Paranagua & China (Shanghai) & 20.50 & 30.50 & 31.00 & 31.50 \\
\hline Rio Grande & China (Shanghai) & 18.00 & 29.50 & 31.00 & 30.70 \\
\hline Santarém & China (Shanghai) & 24.00 & 33.50 & 31.00 & 34.50 \\
\hline São Luís & China (Shanghai) & 23.50 & 30.25 & 31.00 & 33.50 \\
\hline Barcarena & China (Shanghai) & 24.00 & 33.50 & 31.00 & 34.50 \\
\hline
\end{tabular}

*The rates correspond to the average actual values negotiated between shippers and carriers and qtr. = weighted according to the magnitude of the shipped volume.

Note: qtr. = quarter.

Source: University of São Paulo, Escola Superior de Agricultura "Luiz de Queiroz," Brazil (ESALQ/ USP) and USDA, Agricultural Marketing Service. 


\section{Brazil Soybean Transportation}

Table 9. Quarterly ocean freight rates for shipping soybeans from selected Brazilian ports to Germany and China (US\$/metric ton)*

\begin{tabular}{|c|c|c|c|c|c|}
\hline Port & Destination & 1st qtr. 2018 & 2nd qtr. 2018 & 3rd qtr. 2018 & 4th qtr. 2018 \\
\hline Santos & Germany (Hamburg) & 27.00 & 25.00 & 24.00 & 25.00 \\
\hline Paranaguá & Germany (Hamburg) & 28.00 & 26.00 & 25.00 & 26.00 \\
\hline Rio Grande & Germany (Hamburg) & 28.00 & 26.00 & 25.00 & 26.00 \\
\hline Santarém & Germany (Hamburg) & 25.00 & 22.90 & 22.50 & 23.00 \\
\hline São Luís & Germany (Hamburg) & 21.00 & 19.10 & 18.50 & 19.00 \\
\hline Barcarena & Germany (Hamburg) & 23.00 & 20.90 & 20.20 & 20.00 \\
\hline Santos & China (Shanghai) & 32.50 & 31.00 & 27.75 & 30.00 \\
\hline Paranagua & China (Shanghai) & 32.00 & 32.00 & 28.75 & 31.00 \\
\hline Rio Grande & China (Shanghai) & 33.00 & 31.50 & 28.25 & 31.50 \\
\hline Santarém & China (Shanghai) & 38.50 & 35.50 & 31.25 & 34.00 \\
\hline São Luís & China (Shanghai) & 37.00 & 34.80 & 30.75 & 33.00 \\
\hline Barcarena & China (Shanghai) & 37.50 & 33.80 & 32.25 & 35.00 \\
\hline Port & Destination & 1st qtr. 2019 & 2nd qtr. 2019 & 3rd qtr. 2019 & 4th qtr. 2019 \\
\hline Santos & Germany (Hamburg) & 23.00 & 21.50 & 27.00 & 31.00 \\
\hline Paranaguá & Germany (Hamburg) & 23.00 & 21.25 & 27.00 & 30.75 \\
\hline Rio Grande & Germany (Hamburg) & 23.00 & 21.25 & 27.00 & 31.25 \\
\hline Santarém & Germany (Hamburg) & 21.00 & 20.25 & 25.92 & 26.50 \\
\hline São Luís & Germany (Hamburg) & 18.00 & 17.10 & 22.77 & 23.50 \\
\hline Barcarena & Germany (Hamburg) & 19.00 & 17.85 & 23.52 & 24.25 \\
\hline Santos & China (Shanghai) & 32.25 & 30.92 & 33.25 & 38.17 \\
\hline Paranagua & China (Shanghai) & 33.75 & 31.42 & 34.75 & 39.50 \\
\hline Rio Grande & China (Shanghai) & 31.58 & 30.25 & 34.25 & 39.67 \\
\hline Santarém & China (Shanghai) & 32.25 & 30.58 & 38.25 & 39.17 \\
\hline São Luís & China (Shanghai) & 31.00 & 30.58 & 38.25 & 39.42 \\
\hline Barcarena & China (Shanghai) & 32.25 & 29.92 & 38.25 & 39.42 \\
\hline Port & Destination & 1st qtr. 2020 & 2nd qtr. 2020 & 3rd qtr. 2020 & 4th qtr. 2020 \\
\hline Santos & Germany (Hamburg) & 29.25 & & & \\
\hline Paranaguá & Germany (Hamburg) & 30.00 & & & \\
\hline Rio Grande & Germany (Hamburg) & 29.50 & & & \\
\hline Santarém & Germany (Hamburg) & 25.00 & & & \\
\hline São Luís & Germany (Hamburg) & 22.25 & & & \\
\hline Barcarena & Germany (Hamburg) & 24.00 & & & \\
\hline Santos & China (Shanghai) & 35.50 & & & \\
\hline Paranagua & China (Shanghai) & 37.25 & & & \\
\hline Rio Grande & China (Shanghai) & 37.00 & & & \\
\hline Santarém & China (Shanghai) & 36.50 & & & \\
\hline São Luís & China (Shanghai) & 36.75 & & & \\
\hline Barcarena & China (Shanghai) & 38.50 & & & \\
\hline
\end{tabular}

*The rates correspond to the average actual values negotiated between shippers and carriers and qtr. = weighted according to the magnitude of the shipped volume.

Note: qtr. = quarter.

Source: University of São Paulo, Escola Superior de Agricultura “Luiz de Queiroz," Brazil (ESALQ/ USP) and USDA, Agricultural Marketing Service. 


\section{Brazil Soybean Transportation}

\section{Contact Information:}

Delmy L. Salin

Senior Economist, Project Manager

delmy.salin@usda.gov

202.720.0833
Jessica Ladd

Visual Information Specialist

jessica.ladd@usda.gov

202.720.6494

\section{Data Sets (XLS files):}

- Figure 3. Truck rates for selected southern Brazilian soybean export transportation routes, 2017-20

- Figure 4. Truck rates for selected north, south, and northeastern Brazilian soybean export transportation routes, 2017-20

- Figure 5. Brazilian soybean export truck transportation weighted average prices, 2017-20

- Table 1. Quarterly costs of transporting Brazilian soybeans from the southern ports to Shanghai, China

- Table 1a. Quarterly costs of transporting Brazilian soybeans from the northern and northeastern ports to Shanghai, China

- Table 2. Quarterly costs of transporting Brazilian soybeans from the southern ports to Hamburg, Germany

- Table 2a. Quarterly costs of transporting Brazilian soybeans from the northern and northeastern ports to Hamburg, Germany

- Table 3. Quarterly costs of transporting Brazilian soybeans from the southern ports to Shanghai, China

- Table 4. Quarterly costs of transporting Brazilian soybeans from the southern ports to Hamburg, Germany

- Table 5. Quarterly costs of transporting Brazilian soybeans from the northern and northeastern ports to Shanghai, China

- Table 6. Quarterly costs of transporting Brazilian soybeans from the northern and northeastern ports to Hamburg, Germany

- Table 7. Quarterly truck rates for selected Brazilian soybean export transportation routes, 2020

- Table 8. Monthly Brazilian soybean export truck transportation cost index

- Table 9. Quarterly ocean freight rates for shipping soybeans from selected Brazilian ports to Germany and China (US\$/metric ton)

Subscription Information: Send relevant information to GTRContactUs@usda.gov for an electronic copy.

\section{Related Websites:}

- Soybean Transportation Guide: Brazil 2018 (PDF)

- Prior Articles: Brazil Soybean Transportation

- Related Articles: Grain Transportation Report: March 21, 2019 (PDF)

\section{Preferred Citation:}

Salin, Delmy. Brazil Soybean Transportation. May 2020. U.S. Department of Agriculture, Agricultural Marketing Service. Web. <http://dx.doi.org/10.9752/TS052.05-2020>

Photo Credit: USDA

USDA is an equal opportunity provider, employer, and lender. 